\title{
Methods and Procedures Applied to Design of Production Processes and Systems
}

\author{
Naqib Daneshjo ${ }^{1}$, Vladimír Rudy ${ }^{2}$, Peter Drábik ${ }^{1}$, Peter Malega ${ }^{2}$ \\ ${ }^{1}$ Faculty of Commerce of the University of Economics in Bratislava University, Dolnozemská cesta 1, \\ 85235 Bratislava 5, Slovak Republic \\ ${ }^{2}$ Technical university in Kosice, Faculty of Mechanical Engineering, Letná 9, 04001 Košice, Slovak Republic
}

\begin{abstract}
This paper is oriented to the production systems of a company that have to show increased flexibility and a permanent ability in dealing with dynamic requirements of the market and with respect to the products, their functions and variants. Innovative processes and a general support of innovative culture are the key to development and they are an important prosperity tool. They are accompanied by activities that lead to successful production and use of technical novelties and knowledge.
\end{abstract}

Keywords - production systems, modern manufacturing, flexibility, modelling.

\section{Introduction}

Development of productive resources leads to failure-free machines, with the ability to autodetect failures that are simply controlled, quickly adjusted, and highly automatized. The problem of modifying current and future production could be described through many attributes, but the most important aspect is achieving transformation into a knowledgeable society and instilling a new culture of manufacturing. Priority is production that adds the highest value and appropriate knowledge based on

DOI: $10.18421 /$ TEM94-15

https://doi.org/10.18421/TEM94-15

Corresponding author: Daneshjo Naqib,

University of Economics in Bratislava Faculty of

Commerce, Slovak Republic.

Email: daneshjo47@gmail.com

Received: 04 September 2020.

Revised: 14 October 2020.

Accepted: 22 October 2020.

Published: 27 November 2020.

(cc) BY-NC-ND (C) 2020 Daneshjo Naqib et al; published by UIKTEN. This work is licensed under the Creative Commons Attribution-NonCommercial-NoDerivs 4.0 License.

The article is published with Open Access at www.temjournal.com engineering (original products, new productive technologies, new production systems, new approaches to production organization and management). Currently, a complete range of different strategies exist describing development of new products which are implemented in industrially advanced countries.

From the basic methods that can help developing modern manufacturing, the most important ones are those that of greatly accelerate innovation cycles and enable faster and more efficient implementation of process of modernization. How to set the company on the path of optimal strategic position and to preserve its competitiveness on the globalized and crisis-prone market of today is a dilemma of many company owners and managers. The first dilemma is a discrepancy between the economy of scale and the economy of range. The production system is designed for a large scale of outputs without differences in the product design (supporting lower production costs and higher level of automation), or for highly individual products (based on the flexibility of processes and dynamics of working conditions), whereas the second option represents reversal strategies of value and planning orientation. Value orientation focuses on the value added to processes and avoids loses such as planning, preparation, controlling and transfer of processes. Orientation on planning focuses on a large scale planning with the aim to optimize the value added to the process with the support of designing, simulation and information harvesting.

\section{Symbolic Model Generation - Production Processes and Systems}

Various types of modelling have passed through several stages of theoretical development and practical verification, and they are currently used in numerous design activities. Various types of engineering activities models are known to describe objects and processes at the stage of their creation. Particularly useful are the models that enable management of engineering activities through the support of computers [2], [4]. 
Symbolic modelling uses a system of formalized characters - agreed symbols, codes, and parametric data to display objects and processes at the stage of their creation. Issues that arise from the general use of geometric characters, codes, parameters, and their grouping across different users are overcome by standardization. Formalized characters and their models represent an effective language of design and information communication. Application is possible at both the conceptual and partial optimization design level [2]. By integration of methodologies and tools for generating formalized characters and the principles of variant solution of design tasks, interactive, communication methods, computer and information technologies, an efficient simulation system for verifying the concepts of future production variants and their implementation systems, is achieved. In terms of practical procedures, the methodology of modelling through symbolic models should be applied in stages:

1. Analysis of the production process and establishment of a symbolic model of the functional activity of the production system based on system synthesis. Generally, it is necessary to compile several variants of symbolic models that correspond to the expected variants of the production system operation.

2. Optimization of symbolic models is based on a set of optimization criteria. By optimization, more effects can be achieved:

- Bringing the number of stages of a change in the object of production in the production process to minimum.

- Bringing the number of operators in changes of a stage in the object of production to minimum.

- Bringing the current production time to minimum.

- Assessing the assumed complexity of the production system.

- Parameterization of models through additional methods and tools.

Detailed operation of production systems, interpreted through symbolic models, can be based on graphs of the function of motion by the means of production through the reference space, time charts, etc. The methodological procedure of generating models of production processes and their implementation systems through graphic symbols is divided into four levels:

- At the first level, a conceptual model of the production process solution is created. The conceptual model expresses the principle diagram of the production process and establishes the composition and the sequence of the product implementation stages. At this level of modelling, it is advisable to create several variants of symbolic functional structures of the production process, especially when the optimal sequence of partial process implementation is a condition of the efficiency of the whole solution. The graphic presentation of the conceptual model in given case illustrates the deployment of production workstations and systems that effect partial or integral parts of the production process and material flows. Information flows, namely in designing automated production, are modelled on a similar principle. Created models of conceptual nature can be expressed in the form of graphs and may also be drawn as a layout or spatial plans, consisting of layout requirements of production workstations and systems. As an information tool for generating variants of conceptual symbolic models, reference solutions and type-specific design can be used if they are contained in the relevant information systems.

- At the second level, the models of manufacturing operations (procedures) and models of functional operation of production workstations and systems are created. The models deal with detailed composition and sequence of the actions (functions) performed in each operation and the functional activity at the workplace or in the system. If, in addition to the functional, also the technical concept is being resolved, technical units and means are also specified. The breakdown of functional activity in the given case can be performed down to a microelement level corresponding to the movement of the implementation elements or units. Apart from description of designed operations (functional activity), the utility of the modelling also demonstrates in microstructural analyses of classical systems with human service, or in establishing functional sequences of technological and handling operations and their synchronization in automated production. As a result of this modelling stage, there are again several variants of the solution, from which the optimal one is chosen as decision made on the method of optimization.

- At the third level, the model of parametric description is implemented. At this solution level, the respective quantitative parameters (time characteristics, ranges, movement speed, etc.) or qualitative parameter codes are assigned to individual functions expressed by a symbolic character from geometry. A prerequisite is that these parameters are specified in the previous stages of designing a solution, or that they are part of the information system used in the reference patterns.

At the fourth level, spatial and time charts are prepared.

These express the spatial and motion functions performed by the means implemented to that end, as well as the corresponding time dependencies. An example of how a production procedure model is described in symbolic terms is shown in Fig. 1. 


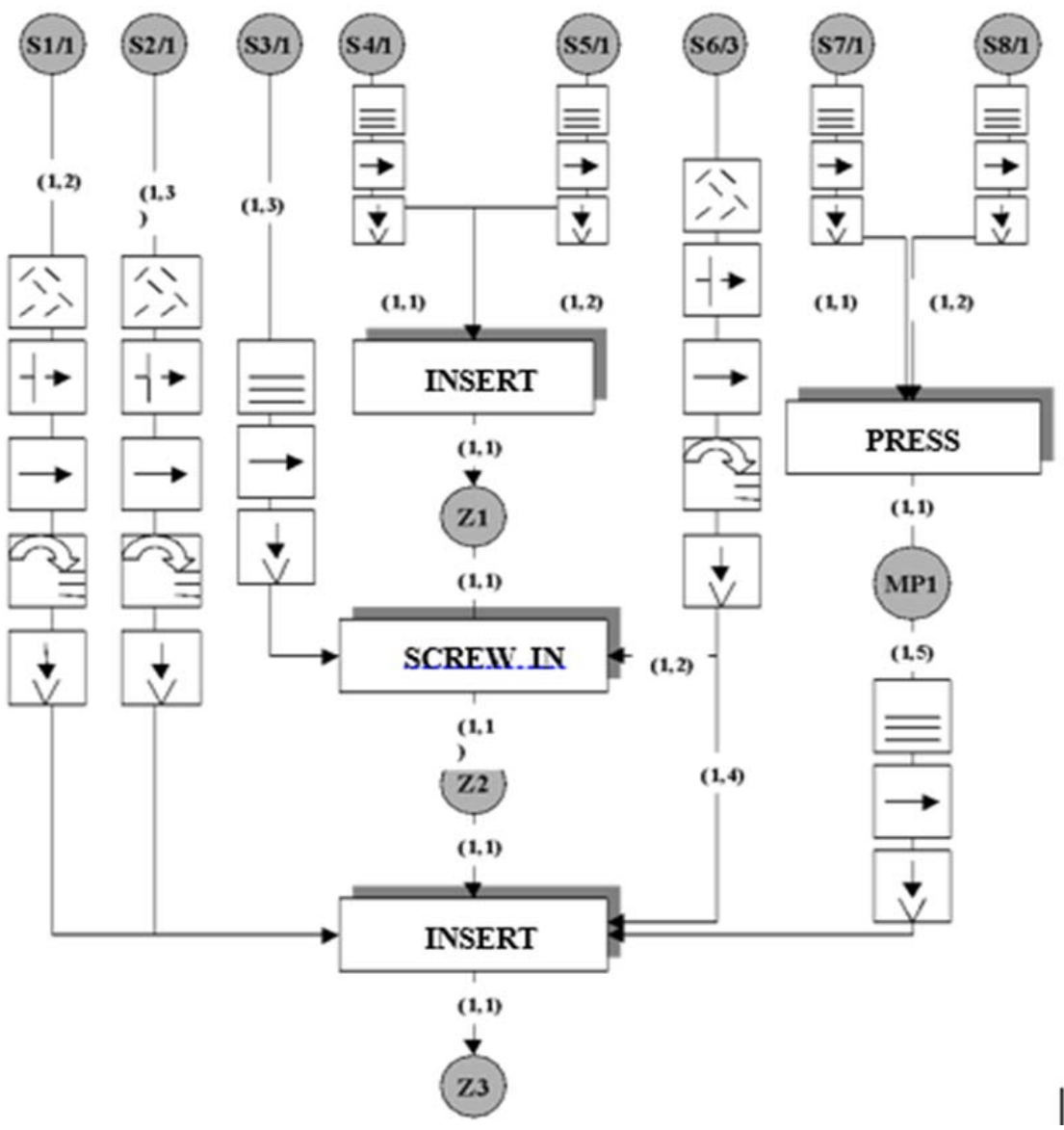

Figure 1. Assembly procedure model using symbolic terms

\section{Mathematical Modelling of Production System Structures}

To increase the efficiency of scientific and technological development, the engineering works have to be automated. In spite of widely used systems of automated design, technological preparation of production, etc. (CAD, CAP, CAx, and other) in specialized areas, further purpose-built development is necessary in this area. Mathematical modelling of the problems, which are to to be solved is an important stage in development of program products. The general benefits of creating mathematical models of objects are also reflected in the benefits of modelling the structures of production systems [3]. The advantages of mathematical modelling are as follows:

- It is applicable to designing production system structures.

- It is suitable for automated systems, but also for human-serviced systems or a combination thereof.
- It increases modelling accuracy compared to most other methods applied so far.

- It conforms with methodological procedures:

a) When creating model databases:

- Objects of production.

- Elements of production systems.

- structures of production systems

b) When using parametric model databases:

- Objects of production.

- Elements of production systems.

- Structures of production systems.

Models of topological relations regarding the means of production in production systems are suitable for solving and optimizing deployment problems. They are characterized by considerable versatility in terms of application conditions, as they are suitable for designing both automated and classic systems [5], [7].

Fig. 2 specifies the basic topological relations of the deployed construction elements of the production system arranged in line. 


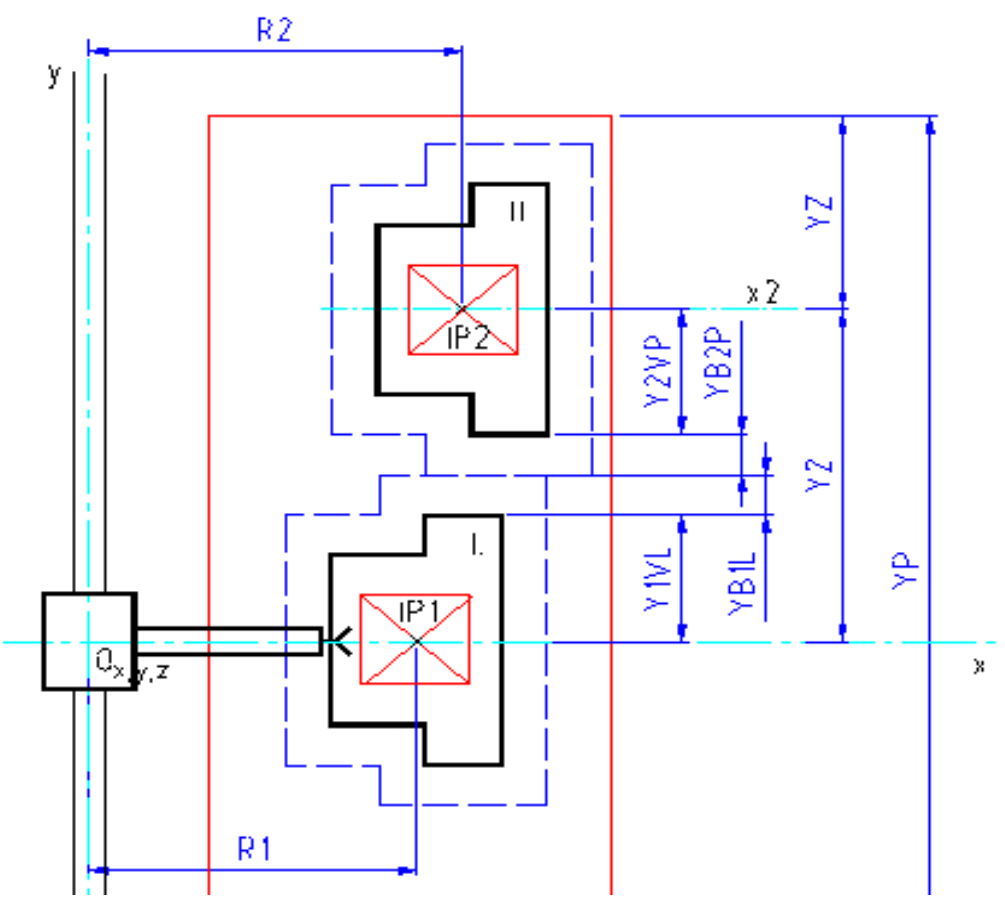

Figure 2. Model of topological relations in production systems arranged in line

The first building element is placed in the working zone at the $I P_{1}$ point $=\left[R_{1}, 0,0\right]$, i.e. on the $x$ axis at the $R_{1}$ distance from the beginning of the coordinate system. $R_{1}$ is determined from the interval

$$
<R_{1 \min }, R_{1 \max }>\text {. }
$$

The second element is placed in the $\mathrm{IP}_{2}$ point $=\left[R_{2}\right.$, $Y 2,0] . R_{2}$ is determined similarly to previous cases (it is recommended to choose $R_{2}=R_{1}$ ). The $Y_{2}$ coordinate is determined as follows:

For an element inserted into the L-half-space:

$$
Y_{2}=Y_{1 V L}+Y_{B I-2}+Y_{2 V P}
$$

The safe distance $Y_{B 1-2}$ is determined as the sum of the safe distances $Y_{1 B L}$ and $Y_{2 B P}$ :

$$
Y_{B 1-2}=Y_{1 B L}+Y_{2 B P /}
$$

The residual distance $\mathrm{Y}_{\mathrm{ZL}}$ is calculated from:

$Y_{Z L}=Y_{P} / 2-Y_{2}-Y_{2 V L}$

For an element inserted into the P-half-space, the following holds true:

$$
Y_{2}=Y_{1 V P}+Y_{B I-2}+Y_{2 V L}
$$

Where:

$Y_{B 1-2}=Y_{1 B P}+Y_{2 B L}, \quad Y_{Z P}=Y_{P} / 2-Y_{2}-Y_{2 V P}$

For $i$ - element in the selected half-space holds true $Y i=Y_{1-1 V}+Y_{B(i-1)-1}+Y_{i V}$,

Where:

$Y_{B / i-1)-1}=Y_{i-1 B}+Y_{i B}, Y_{Z}=Y_{P} / 2-Y i-Y_{i V}$
Where:

For L-half- space:

$i=i_{L}$

$$
\begin{aligned}
& Y_{i-l V}=Y_{i-1 V L} \\
& Y_{i V}=Y_{i V P} \\
& Y_{i-1 B}=Y_{i-1 B L} \\
& Y_{i B}=Y_{i B P} \\
& Y_{Z}=Y_{Z L} \\
& Y_{i V^{\prime}}=Y_{i V L}
\end{aligned}
$$

For $P$-semi-master $i=i P$ :

$$
\begin{aligned}
& Y_{i-I V}=Y_{i-1 V L} \\
& Y_{i V}=Y_{i V P} \\
& Y_{i-1 B}=Y_{i-1 B L} \\
& Y_{i B}=Y_{i B P} \\
& Y_{Z}=Y_{Z L} \\
& Y_{i V^{\prime}}=Y_{i V L}
\end{aligned}
$$

When applying the given procedure to elements of relatively complex shapes, there may be a case when a spare distance y $\mathrm{R}$ remains between $\mathrm{i}-1$ and i-th element, which can further reduce the distance between the relevant elements. Correction of $i$-th element's position is affected by reducing the coordinate $\mathrm{y}_{2}$ by the correction distance $y_{R}$.

The possibility of correcting the distance in the critical points of the elements is examined (critical points are considered to impart a significant change in the external shape of the element). 


\section{Designing Catalogue of Production Systems}

Information for the purpose of designing production systems is required in routine, comprising creative, technical and engineering activities. Such information should be available, highly up-to-date and reasonable. Large diffusion of information, its amount and other aspects cause searching, processing and using problems. For the purpose of designing, comprehensive and rational information systems need to be developed and implemented.

They should include not only literature and documentary data, but also useful factual and graphic information. Design information systems are subject to the following requirements:

- They should collect, process, store and supply different types of professional knowledge and information (factual and graphic) as needed.

- Information can be presented in various forms (numerical, verbal, and graphic).

- The partial information modules generated from the information system are relatively independent and compatible with each another in terms of integration.

- In the process of solving a design task, information can be selected from various partial modules.

- The core of the specific differences between the documentary and graphic, the factual and graphic system, is in accessing of set of document records that are relevant to information question (information documents).

A designer's own methodological procedures and algorithms of their work for creating type-specific designs are based on interactive principle of the designer's work and on their creativity. The designer decides not only on the manner, the methods and techniques of the solution, but they use the computer system and its software and information equipment as an intelligent tool. Through the offered selection of possible activity variants, the designer is able to feed the process with data for it to supply a solution at any stage [1], [6]. The whole principle of interactive designer inputs is the same as the so called - "Environmentally controlled events" (interface). The selection methods applied are an important part of information systems. Through them, information is selected from an appropriate database.

The following selection methods are suitable for the information system:

- Direct selection. It allows selecting relevant factual and graphic information according to type designation, password, or code. In this case, the name of the selection function is the same as type designation of the information file.

- Parametric selection. Selection of information files according to defined set of factual and graphic parameters. The demand vector is compiled and based on weight criteria, or the main criterion and the associated restrictions.

- Selection by scanning. A classic way of selection, comparable to the principle of browsing and searching for information in the classical factual and graphic catalogue.

- Expert selection. Expert knowledge in decisionmaking activities is used. The general approach is based on generating variants, setting evaluation criteria, evaluating and choosing the optimal variant.

Type designs need to be expressed in design documentation in such a way as to ensure technical feasibility of their implementation under the given conditions.

An important factor of design documentation creation is the uniqueness of the solution and the completeness of the information. It is also necessary to consider that the methodological design procedure and the content of design documentation are generally legally enshrined in relevant regulations [8].

In terms of solution needs, it is recommended to express the structures of type projects in two forms:

1. Catalogues of simplified type designs (topics).

2. Comprehensive design documentation for implementation according to legislative regulations (for selected reference models).

It is necessary to develop a system of uniform forms to record information on type designs (simplified form). From the methodical point of view, recording should be done according to the agreed rules and type principles.

This proposal is based on the hierarchical principle of information and knowledge classification. Basic information units are specified below:

1. Identification part.

2. Spatial (layout) solution.

3. Functional description (characteristics).

4. Structural and technical and economic indicators.

5. Product profile (representatives of product group).

6. Technological profile (reference implementation technologies).

7. Equipment and facilities (units for expanding functionalities).

8. Basic variant modifications.

9. Special supplementary data or documents. 


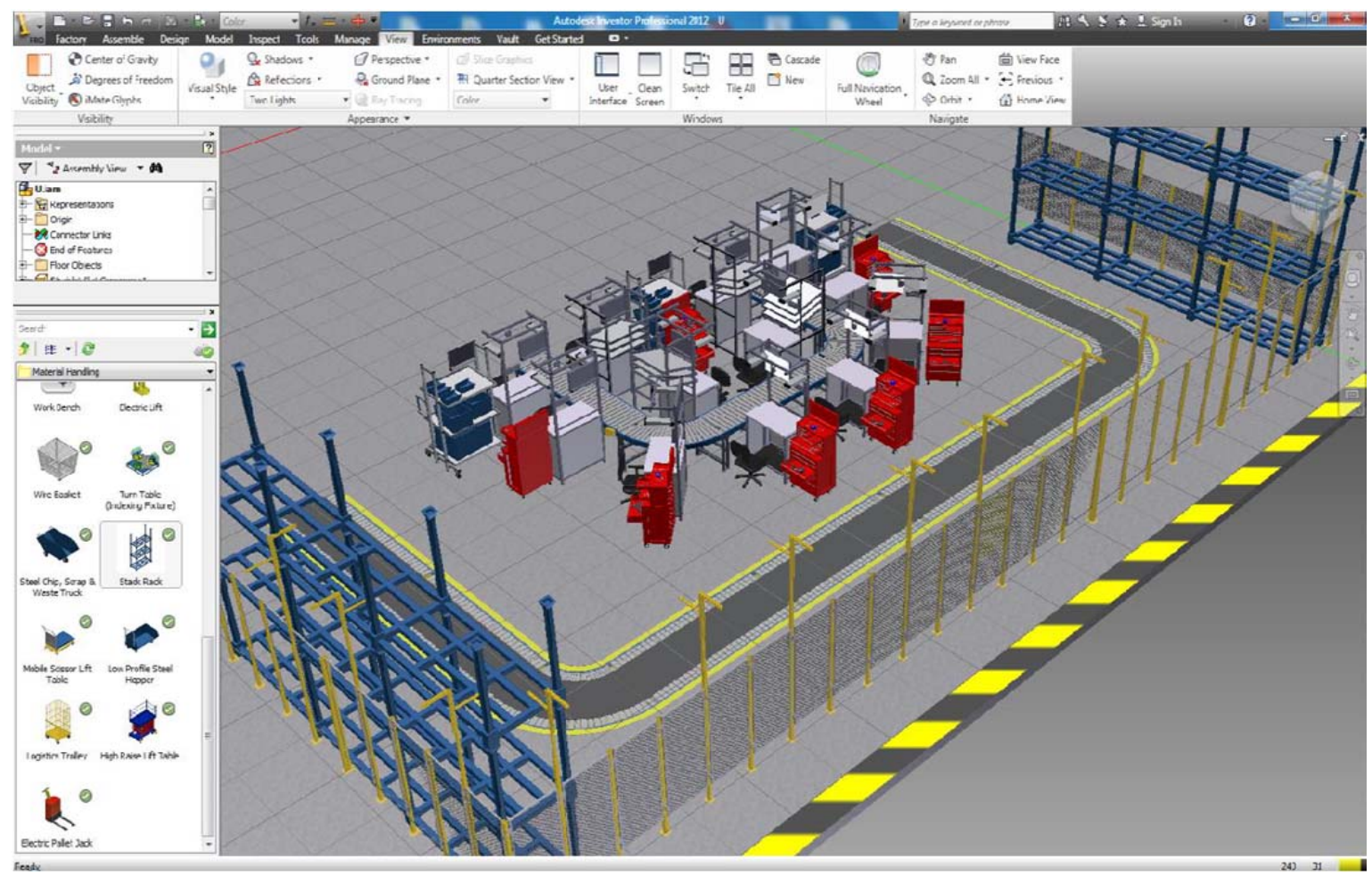

Figure 3. An example of the SW product architecture for building production workstation structures

The SW form of the application product for Fig. 3. An example of the content of manufacturing creating structures of production workstations is in technology database is illustrated in Fig. 4.

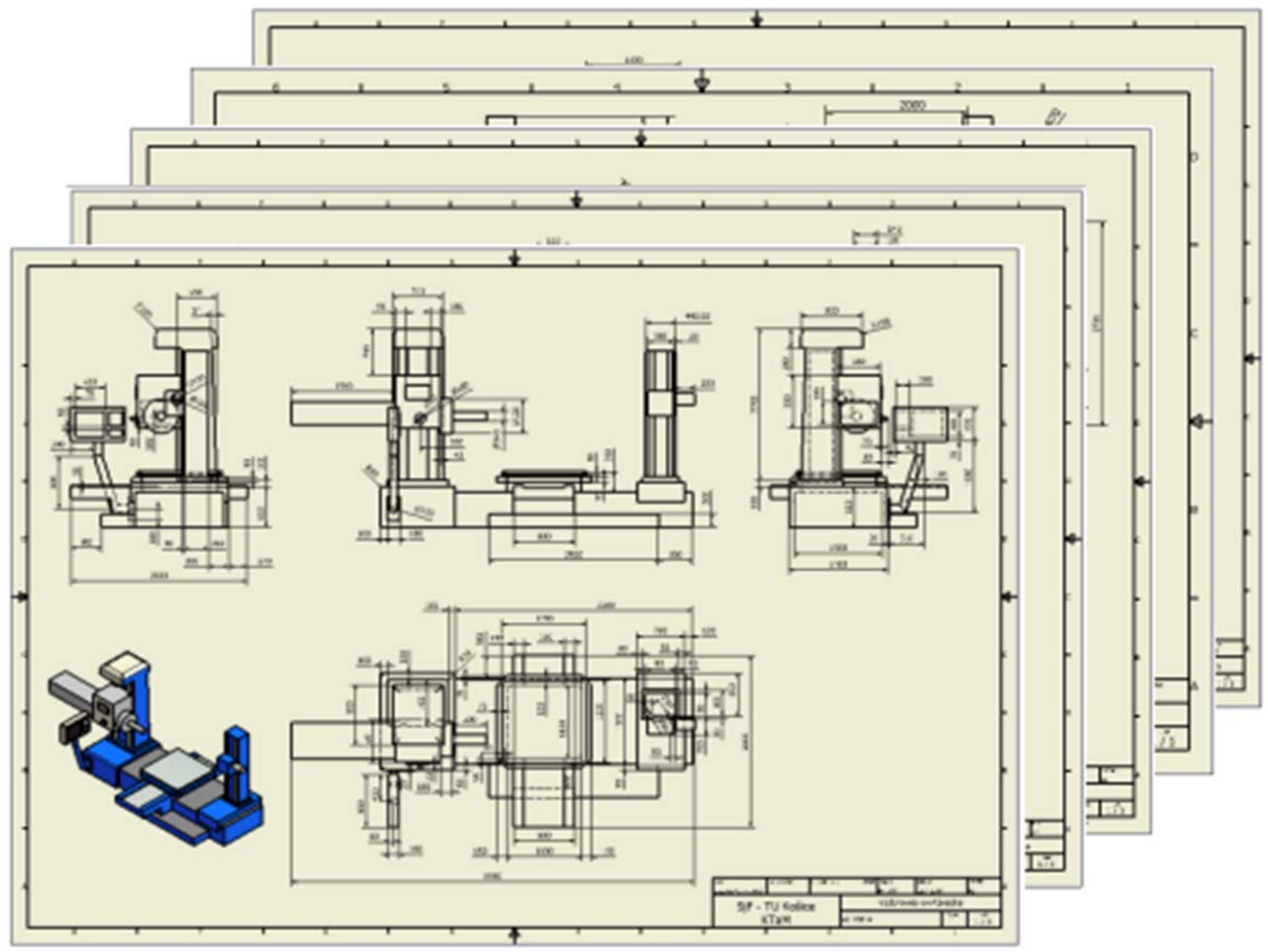




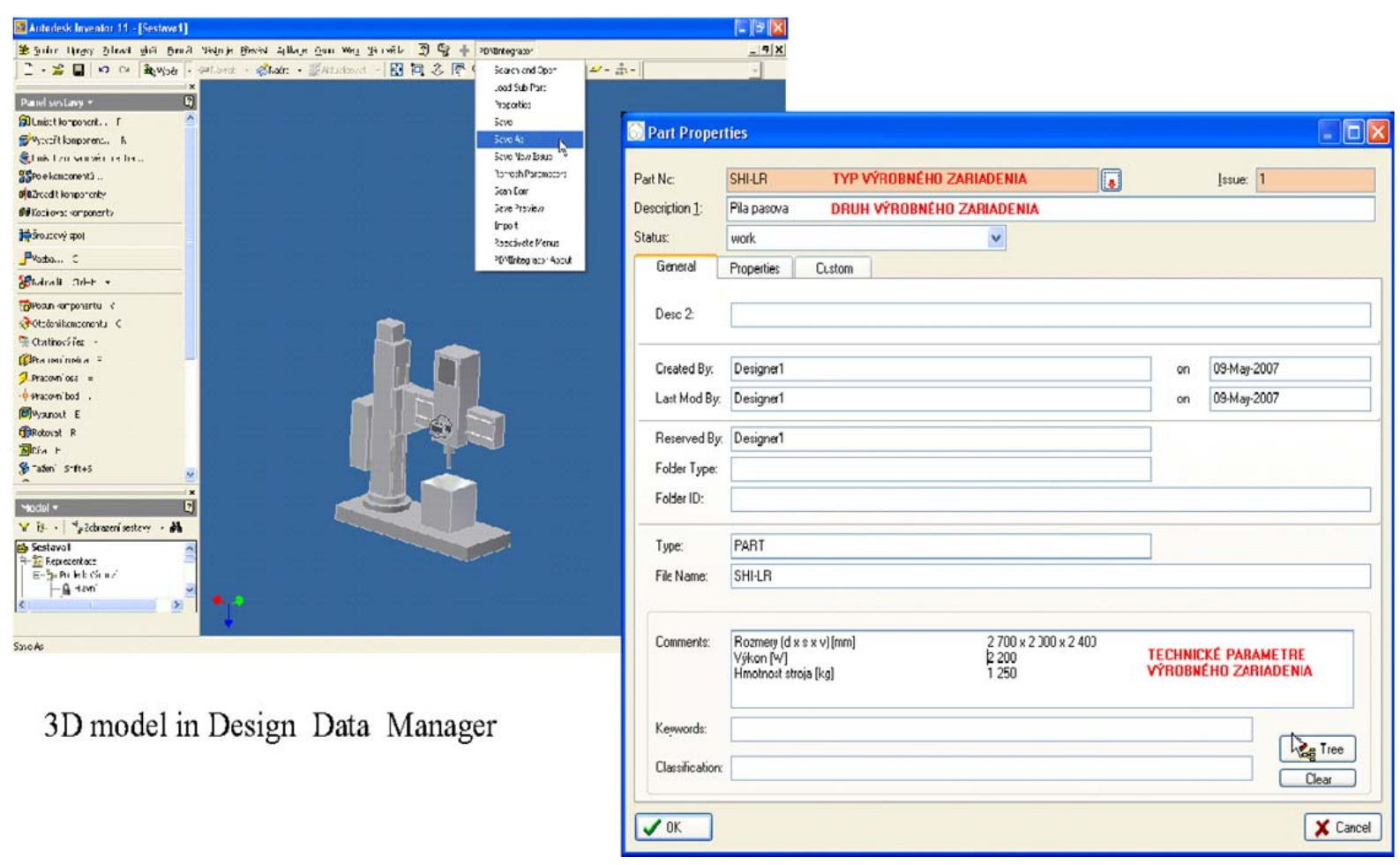

Figure 4. An example of catalog sheet from the production machinery database

Engineering production design requires a system solution for a set of structural, technological, spatial and organizational factors resulting in the design of an optimal manufacturing configuration. The goal is the functional integration of people, information, production technology, materials and energy to prevent wasting these resources, and to achieve high productivity. The efficiency of operation depends on the level of mutual synchronization, the ability to predict and eliminate adverse effects of these factors on the production characteristics of the production system. So the role of design activity is to design and, in the form of design documentation, to process the production configuration that can be executed under optimal financial conditions in the required space and time, capable to meet time-limited market demands.

The final design, while taking into account the mutual synergy of these factors and guaranteeing the consistency of designed ideas with the achieved results, leads in practice to the complexity and variability of possible solutions. Capability of reconfiguration or building new innovative productions is, therefore, one of the most complex tasks in the company life cycle. It requires the application of adequate computer, information and software support for modern CAx systems, which are capable to apply standardized design procedures and to modify configurations from "proven" designs.

Designing today's manufacturing systems requires a comprehensive analysis of their activities in real space and time. Only this approach is a guarantee of identification, optimization, or elimination of design deficiencies before their future physical implementation. It is necessary to take into account the philosophical aspects of technological innovations, modelling, examples and studies of successful reference solutions, financial support and material provision for innovative changes, multipurpose and purpose-oriented designs for the synthesis of technological innovations, etc.

\section{Conclusion}

Concepts of new production systems are currently designed as the systems of new generation. The aim of such solutions is to increase productivity without losing flexibility, shortening production time, increasing the quality and the value of products and services, and so on. They are modified as social and economic systems and place high demands on design, implementation and operation. The numerous papers in professional literature deal with their development [2], [9]. The current market offers application software systems that support manufacturing system design in different application areas. The use of such systems allows designing the entire production system, to purposely situate the means of production in the production area, to design traffic paths, storage areas, to make their simulation, to display and describe them from any views, etc.

Current development enables these different approaches to be included in a single integrated system. Synchronous work in real and virtual environments brings a number of effects. Integrated procedures ensure that all actions applied to real 
physical models are transferred to parallel work with computer models that are subsequently presented in virtual environment. An important innovative method of designing production systems is simulation and its methods, techniques and tools. The construction of production systems based on the pillars of "flexibility / diversity, autonomy, recognition ability" is possible with the help of technologies that could be synergistically functional, and mutually combined. Such technology is the modularity of production technology, robotic technology with exchangeable tools and sensors, multi-sensor integration, data fusion, etc. The robotic technology, together with the standardization elements, plays the key role.

The vision of the adaptive, fast configuring production systems of the future points to subsystems of natural intelligence (the ability of recognition) which communicate together and cooperate in synergy with the predefined target functions. The key role in such systems is the permanent monitoring of the execution of the production target, with the aim of avoiding or minimizing negative effects on quality. The integration of sensing technologies and the fusion of different data sensors is necessary to ensure the quality evaluation of different products and processes. From the currently available IT technologies and hardware components, we can conclude that this autonomous check and synchronization could be implemented to a wide range of technical options which are able to sense the environment, and they are able to make decisions in a target oriented way.

\section{Acknowledgement}

This work has been supported by the Scientific Grant Agency of the Ministry of Education of the Slovak Republic (Project KEGA 032EU-4/2020 a KEGA 002TUKE-4/2020)

\section{References}

[1]. Bučková, M., Krajčovič, M., \& Edl, M. (2017). Computer simulation and optimization of transport distances of order picking processes. Procedia engineering, 192, 69-74.

[2]. Daneshjo, N., Rudy, V., Štofková, K., Mareš, A., Kováč, J., Jahnátek, A., ... \& Rusnák, J. (2018). Inteligent industrial Engineering-Innovation potential. San Antonio: FedEx Print \& Ship Center.

[3]. Hammer, M. (2001). The superefficient company. Harvard business review, 79(8), 82-93.

[4]. Daneshjo N., Kralik, M., \& Danishjoo, E.(2018). Logistical measurement strategy on coordinate measuring machines as a tool for the reproducibility of results. In: MM (Modern Machinery) Science Journal, Czech Republic, March (2018), 2338-2345

[5]. Malega, P., \& Kovac, J. (2016). Design of assembly system--mixed reality modelling. Annals of DAAAM \& Proceedings, 289-298.

[6]. Králik, M., Jerz, V., \& Paštéka, M. (2019, August). Optimization of the Machine and Device Layout Solution in a Specific Company Production. In Proceedings of the International Symposium for Production Research 2019 (pp. 91-103). Springer, Cham.

[7]. Kováč, J., \& Rudy, V. (2014). Innovation production structures of small engineering production. Procedia Engineering, 96, 252-256.

[8]. Semjon, J. (2013). The procedure designing production systems based on CA methods. In Applied Mechanics and Materials (Vol. 282, pp. 246-251). Trans Tech Publications Ltd.

[9]. Wiendahl, H. P., Reichardt, J., \& Nyhuis, P. (2015). Handbook factory planning and design. Springer. 